\title{
A Reactance-Domain Concurrent Blind Beamformer
}

\author{
L. Ortolan, T. L. S. Santos, M. C. F. DeCastro and F. C. C. DeCastro
}

\begin{abstract}
The electronically steerable parasitic array radiator (ESPAR) is a smart antenna suited for low-cost user terminal applications. Beamforming is achieved by adaptively adjusting the load reactances at parasitic elements surrounding the active central element. The main goal is to establish nulls in the interfering directions of the array radiation pattern. This paper presents a new blind beamformer for use with reactance domain controlled arrays, such as ESPAR antennas. The new beamformer is based on the concurrent operation of two stochastic gradient algorithms, one which minimizes a cost function that measures the received signal energy dispersion and other which minimizes the Euclidean distance between the received digital modulation symbols and the ones in the reference constellation. Results show that the new blind beamformer presents a faster convergence and a lower steady state mean squared error when compared with classical blind deconvolution algorithms, such as the Maximum Moment Criterion or the Constant Modulus Algorithm.
\end{abstract}

Index Terms - smart antennas, blind beamforming, concurrent deconvolution.

\section{INTRODUCTION}

A receiving smart antenna consists of a multiple element array whose impinging signals are processed adaptively in order to exploit the spatial dimension of the wireless channel. Specifically, the beamforming action can improve link quality by reducing the intersymbol interference due to multipath effects.

There are two broad classes of beamformers - blind and non-blind. Unlike its counterpart class (e.g. [1]), the class of blind beamformers does not require the transmission of a training sequence in order to minimize a cost function, which is an advantageous feature in terms of spectral efficiency and bandwidth saving. The Maximum Moment Criterion (MMC) [11] has been proposed as a blind beamforming algorithm for ESPAR antennas. Since the MMC cost function measures solely the energy dispersion at the beamformer output - and therefore does not take into account the phase of the received complex valued IQ symbols - even after the MMC convergence the resulting output signal usually presents its

Manuscript received September 2, 2006. This work was supported by the Brazilian Government - FINEP - Financiadora de Estudos e Projetos.

L. Ortolan, T. L. S. Santos, M. C. F. DeCastro and F. C. C. DeCastro is with Wireless Technologies Research Center, Pontifical Catholic University of Rio Grande do Sul, BRA (e-mail: cptw@pucrs.br). constellation rotated with respect to the demodulator reference constellation.

The Concurrent Equalizer (CE) is a blind deconvolution algorithm based on the principle of the concurrent operation between the direct decision (DD) equalizer and the constant modulus (CMA) equalizer [3]. The minimization of the Euclidean distance based DD cost function only takes place when the minimization of the energy dispersion based CMA cost function is judged to have achieved a successful adjustment with high certainty. Certainty is measured as the closeness of the output to the same IQ symbol in the reference constellation before and after a perturbation is imposed to the equalizer. Due to the high performance improvement achieved with this approach, the CE has been considered as a state of the art technique in the context of temporal deconvolution [12].

This work proposes a new blind beamformer based on the concurrent operation of the MMC and DD algorithms, subjected to the same certainty criterion adopted for the Concurrent Equalizer. Simulation results show that the concurrent MMC-DD acting as a beamformer not only presents a better signal to interference ratio than the MMC, as shown in Figure 1, but also results in a lower symbol error rate (SER) at the digital demodulator output.

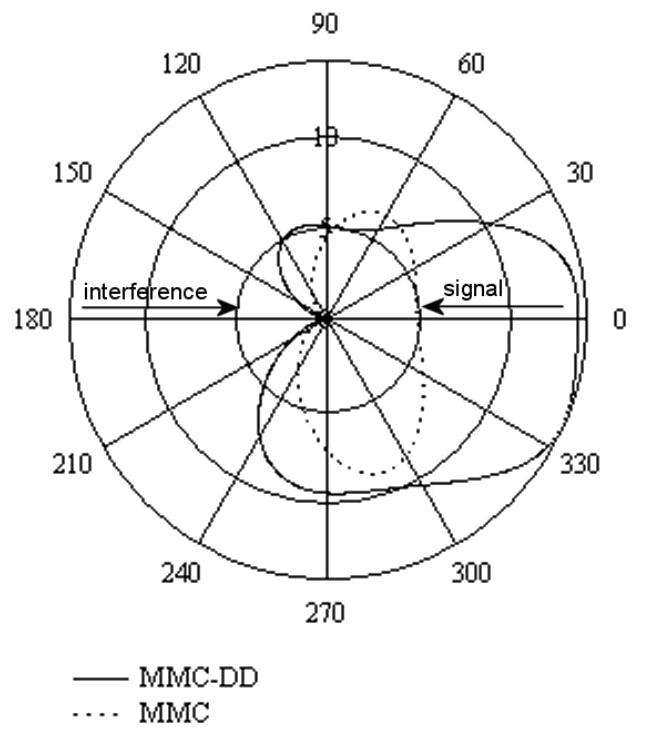

Fig. 1 Array radiation pattern for the MMC and MMC-DD beamformers operating with 16-QAM modulation. The array is composed of 7 half-wave dipoles - 6 passive equally spaced dipoles plus an active central one. 


\section{THE ANALYTIC MODEL OF THE ARRAY}

A ESPAR array is usually composed of 6 quarter-wave passive monopoles located on a ground plane and equally spaced along a circle of radius $\lambda / 4$, being $\lambda$ the operating wavelength [1][2]. Only a central active monopole is connected to the RF front-end of the digital receiver. All the other 6 passive monopoles are parasitic elements which are adjusted by means of a variable reactance $X_{i}, i=1,2, \cdots, 6$ located at the base of the respective monopole. These reactances are the free parameters of the ESPAR which are adjusted by the beamformer algorithm.

In this work, in order to avoid ground plane effects on the array radiation pattern, the quarter-wave dipoles on a ground plane are replaced by a half-wave dipole array, as shown in Figure 2.

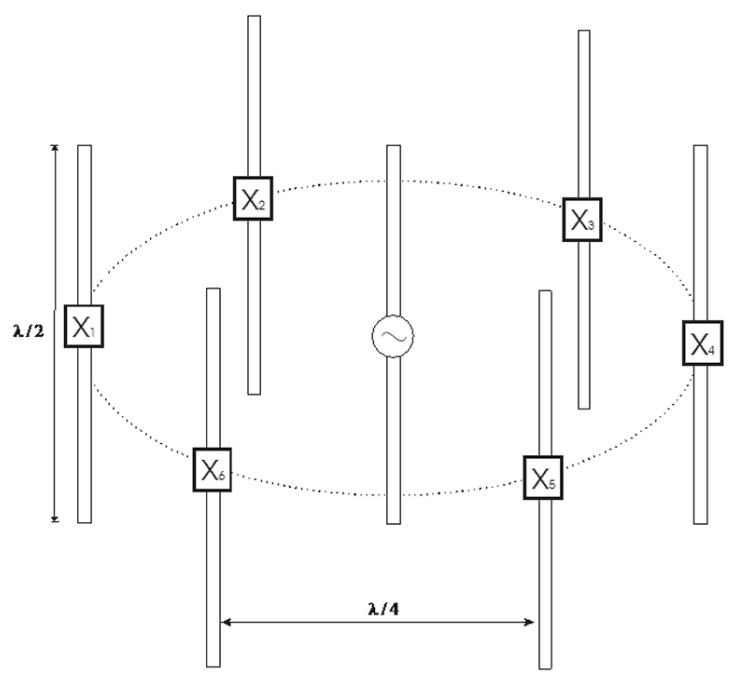

Figure 2 Half-wave dipole array adopted in this work for the assessment of the MMC-DD beamformer performance. The variable reactances $X_{i}$ are placed at the half-length of the respective half-wave dipole.

The ESPAR can be analytically described by a general adaptive array composed of $M$ dipoles of length $\ell$ parallel to $z$ axis, as shown in Figure 3. The $m^{\text {th }}$ dipole is located at coordinates $\left(x_{m}, y_{m}, z_{m}\right), m=0,1 \cdots M-1$.

The $M$ signals $u_{m}$ at each dipole output in the array of Figure 3 are linearly combined by a set of respective complex valued coefficients $w_{m}$, yielding the output signal $r$. Let $\underline{w}=\left[\begin{array}{llll}w_{0} & w_{1} & \cdots & w_{M-1}\end{array}\right]^{T}$ be the vector whose components are the coefficients $w_{m}$. The beamformer adjusts $\underline{w}$ aiming to maximize the quality of the output signal $r$ according to an optimization criterion. In the context of a beamformer for an ESPAR antenna, the adjustment of $\underline{w}$ has an equivalent effect to the adjustment of the ESPAR reactances. Therefore, this analytic approach has been denoted as Equivalent Weight Vector (EWV) [1][2]. As we shall see later, the ESPAR of Figure 2 can be described by the general adaptive array shown in Figure 3 provided the EWV analytic expression is used to determine the coefficients $w_{m}$ and the geometric parameters of Figure 2 are applied.

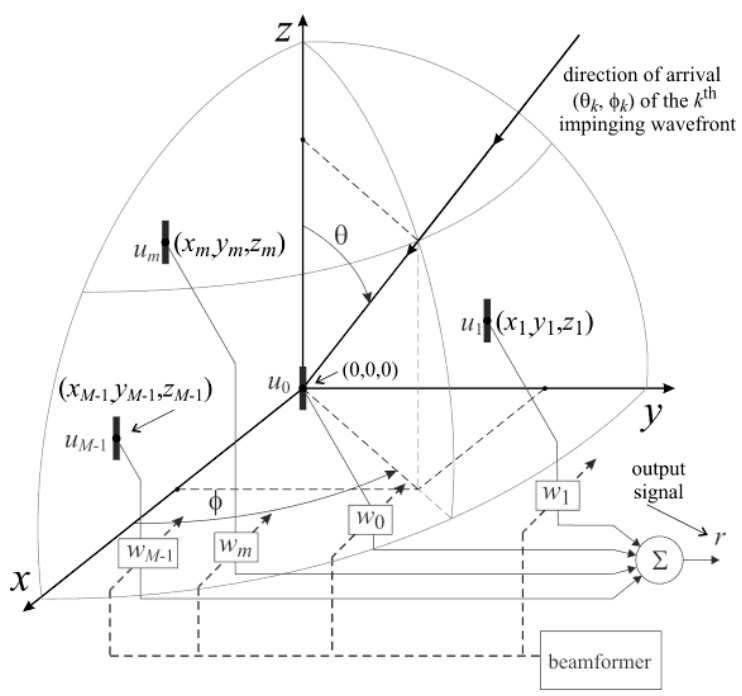

Fig. 3 General adaptive array composed of $M$ dipoles of length $\ell$. The center of the $m^{t h}$ dipole is located at coordinates $\left(x_{m}, y_{m}, z_{m}\right)$.

Figure 3 also shows the $k^{\text {th }}$ wavefront that impinges the array at direction of arrival $\left(\theta_{k}, \phi_{k}\right), \quad k=0,1, \cdots, K-1$, where $K$ is the total number of signals that arrives at the array. Let $s_{k}(n)$ be the $n^{\text {th }}$ sample of the baseband complex envelope received from direction $\left(\theta_{k}, \phi_{k}\right)$. The time interval between samples $n$ and $n+1$ is given by the inverse of the digital modulation symbol rate. The $n^{\text {th }}$ sample $r(n)$ of the array output signal resulting from $K$ impinging wavefronts is given by

$$
r(n)=A \sum_{k=0}^{K-1} s_{k}(n) F\left(\theta_{k}, \phi_{k}\right)
$$

where $A=131.2$ is a gain constant obtained by practical measurement [1], $F(\theta, \phi)$ is the array radiation pattern [4] given by

$$
F(\theta, \phi)=F_{d}(\theta, \phi) f(\theta, \phi)
$$

$F_{d}(\theta, \phi)$ is the radiation pattern of each dipole of length $\ell$ [9] given by 


$$
F_{d}(\theta, \phi)=\frac{\cos \left(\frac{\ell}{\lambda} \pi \cos \theta\right)-\cos \left(\frac{\pi \ell}{\lambda}\right)}{\operatorname{sen} \theta}
$$

$f(\theta, \phi)$ is the array factor [4] given by

$$
f(\theta, \phi)=\underline{w}^{\mathrm{T}} \cdot \underline{a}(\theta, \phi)
$$

where

$$
\underline{w}=\left[\begin{array}{llll}
w_{0} & w_{1} & \cdots & w_{M-1}
\end{array}\right]^{T}
$$

and

$$
\underline{a}(\theta, \phi)=\left[\begin{array}{llll}
1 & a_{1}(\theta, \phi) & \cdots & a_{M-1}(\theta, \phi)
\end{array}\right]^{T}
$$

is the steering vector [4] in direction $(\theta, \phi)$ with $M$ components $a_{m}(\theta, \phi), m=0,1 \cdots M-1$, given by

$$
a_{m}(\theta, \phi)=e^{j \frac{2 \pi}{\lambda}\left(x_{m} \operatorname{sen} \theta \cos \phi+y_{m} \operatorname{sen} \theta \operatorname{sen} \phi+z_{m} \cos \theta\right)}
$$

Notice from (1) and (2) that the output signal $r(n)$ is a function of the array factor $f(\theta, \phi)$ given by (4). On the other hand, $f(\theta, \phi)$ is a function of vector $\underline{w}$, whose components are adaptively determined by the beamformer algorithm. Thus, the beamformer controls the array via adjustment of vector $\underline{w}$. For the analytic description of the ESPAR antenna operation, vector $\underline{w}$ is determined by the EWV expression [1][2][11]:

$$
\underline{w}=(\mathbf{Z}+\underline{X})^{-1} \underline{U}
$$

where $\mathbf{Z}$ is the mutual impedance matrix given by (12), $\underline{X}=\left[\begin{array}{lllllll}50 & X_{1} & X_{2} & X_{3} & X_{4} & X_{5} & X_{6}\end{array}\right]^{T}$ is the reactance vector, with reactance $X_{i}, i=1,2, \cdots, 6$ representing the reactances at the center of each passive dipole controlled by the beamformer and with the $50 \Omega$ resistance representing the RF front end input impedance presented to the active central dipole. Vector $\underline{U}$ represents the normalized voltages at the center of each dipole [2]:

$$
\underline{U}=\left[\begin{array}{lllllll}
1 & 0 & 0 & 0 & 0 & 0 & 0
\end{array}\right]^{T}
$$

Notice that the linear combination of the $M$ signals $u_{m}$ weighted by the respective coefficients $w_{m}$ yielding the output signal $r$ can be expressed as

$$
r(n)=\underline{w}^{\mathrm{T}}(n) \cdot \underline{u}(n)
$$

signals $u_{m}$ at the respective dipole output:

$$
\underline{u}=\left[\begin{array}{llll}
u_{0} & u_{1} & \cdots & u_{M-1}
\end{array}\right]^{T}
$$

The mutual coupling between the array elements affects the radiation pattern sidelobes after the beamformer convergence [5]. In this work the mutual coupling effects are modeled by the Method of Moments (MoM) [6][7] with triangular basis functions [8] and weighting of residuals [10]. Specifically, the mutual impedance $z_{i j}$ between the $i^{\text {th }}$ and $j^{\text {th }}$ dipoles of a 7 element ESPAR is defined by the array mutual impedance matrix $\mathbf{Z}$ [2]:

$$
\mathbf{Z}=\left[\begin{array}{lllllll}
z_{00} & z_{01} & z_{02} & z_{03} & z_{04} & z_{05} & z_{06} \\
z_{10} & z_{11} & z_{12} & z_{13} & z_{14} & z_{15} & z_{16} \\
z_{20} & z_{21} & z_{22} & z_{23} & z_{24} & z_{25} & z_{26} \\
z_{30} & z_{31} & z_{32} & z_{33} & z_{34} & z_{35} & z_{36} \\
z_{40} & z_{41} & z_{42} & z_{43} & z_{44} & z_{45} & z_{46} \\
z_{50} & z_{51} & z_{52} & z_{53} & z_{54} & z_{55} & z_{56} \\
z_{60} & z_{61} & z_{62} & z_{63} & z_{64} & z_{65} & z_{66}
\end{array}\right]
$$

where $\mathbf{Z}$ is a discretized solution of the retarded potential integral equation for the unknown current I in the $i^{\text {th }}$ dipole subjected to the condition that the scattered electric field generated by I cancels the total impressed electric field at the surface of the $j^{\text {th }}$ dipole [13][14].

The ESPAR geometric symmetry [2] results in the following relationships for impedances $z_{i j}$ :

$$
\begin{aligned}
& z_{11}=z_{22}=z_{33}=z_{44}=z_{55}=z_{66} \\
& z_{01}=z_{02}=z_{03}=z_{04}=z_{05}=z_{06} \\
& z_{12}=z_{23}=z_{34}=z_{45}=z_{56}=z_{61} \\
& z_{13}=z_{24}=z_{35}=z_{46}=z_{51}=z_{62} \\
& z_{14}=z_{25}=z_{36}
\end{aligned}
$$

Based on the array geometry shown in Figure 2, the impedances $z_{i j}$ determined by the MoM are:

$$
\begin{aligned}
& z_{00}=85.00+j 48.60 \\
& z_{11}=85.00+j 48.60 \\
& z_{01}=43.28-j 37.12 \\
& z_{12}=43.00-j 36.65 \\
& z_{13}=5.38+j 40.23 \\
& z_{14}=17.80+j 32.02
\end{aligned}
$$




\section{THE CONCURRENT MMC-DD BLIND BEAMFORMING PROCEDURE}

In order to perform the concurrent minimization of the MMC and DD cost functions, this work follows the heuristic presented in [2], which consists of perturbing the reactance components $X_{i}$ of vector $\underline{X}, i=1,2, \cdots, 6$, at each iteration $n$ so that the cost functions gradient can be instantaneously estimated. At the beamformer startup vector $\underline{X}$ is initialized as $\underline{X}(n=0)=\left[\begin{array}{lllllll}50 & 0 & 0 & 0 & 0 & 0 & 0\end{array}\right]^{T}[\Omega]$.

Let $\underline{X}_{\text {up }}$ and $\underline{X}_{\text {down }}$ be the vectors which result from perturbing $\underline{X}$ in opposed directions:

$$
\begin{aligned}
& \underline{X}_{\text {up }}(n)=\underline{X}(n)+c(n) \underline{\Delta}(n) \\
& \underline{X}_{\text {down }}(n)=\underline{X}(n)-c(n) \underline{\Delta}(n)
\end{aligned}
$$

with $c(n)$ being the variable which 0controls the amount of perturbation applied at iteration $n$ :

$$
c(n)=\frac{C}{(n+1)^{\gamma}}
$$

where $C$ and $\gamma$ are constant nonnegative arbitrary parameters

$\Delta(n)=\left[\begin{array}{lllllll}0 & \delta_{1}(n) & \delta_{2}(n) & \delta_{3}(n) & \delta_{4}(n) & \delta_{5}(n) & \delta_{6}(n)\end{array}\right]^{T}$ is a vector whose components $\delta_{i}(n), \quad i=1,2, \cdots, 6$, randomly take on values from the set $\{1,-1\}$ with uniform probability [2]. Notice that $c(n)$ is monotonically decreasing as a function of the iteration index $n$, thus, avoiding local minima at the beginning of the gradient descent optimization.

The MMC algorithm cost function [2][11] is

$$
L(n)=1-|M M C(\underline{r}(n), N a)|^{2}
$$

where vector $\underline{r}(n)=\left[\begin{array}{lllll}r\left(n-N_{L}+1\right) & \cdots & r(n-1) & r(n)\end{array}\right]^{T}$ represents a buffer which stores the last $N_{L} \geq N a$ samples $r(n-k)$ of the array output signal, $k=0,1 \cdots, N_{L}-1$, and $M M C(\underline{r}(n), N a)$ is given by

$$
M M C(\underline{r}(n), N a)=\frac{\left(\frac{1}{N a} \sum_{k=0}^{N a-1}|r(n-k)|\right)^{2}}{\left.\frac{1}{N a} \sum_{k=0}^{N a-1}(\mid r(n-k))\right)^{2}}
$$

where $N a$ is the number of averaged samples in (17).

Since the MMC cost function $L$ depends on the reactance vector $\underline{X}$, then from (13), (14) and (16) we can define

$$
\begin{gathered}
L_{\text {up }}(n)=L\left(\underline{X}_{\text {up }}(n)\right) \\
L_{\text {down }}(n)=L\left(\underline{X}_{\text {down }}(n)\right)
\end{gathered}
$$

Thus the gradient of $L$ can be expressed as

$$
\begin{gathered}
\underline{\nabla_{L}(n)=\frac{\Delta L(n)}{\Delta \underline{X}(n)}}= \\
{\left[\begin{array}{llllllll}
0 & g_{1}(n) & g_{2}(n) & g_{3}(n) & g_{4}(n) & g_{5}(n) & g_{6}(n)
\end{array}\right]^{T}}
\end{gathered}
$$

where $g_{i}(n), \quad i=1,2, \cdots, 6$, are components of the gradient given by

$$
g_{i}(n)=\frac{L_{\mathrm{up}}(n)-L_{\mathrm{down}}(n)}{2 c(n) \delta_{i}(n)}
$$

Once gradient $\underline{\nabla_{L}}$ is obtained, a first update based on $\underline{\nabla_{L}}$ is applied to vector $\underline{X}$ :

$$
\underline{X^{\mathrm{I}}}(n)=\underline{X}(n)-a(n) \underline{\nabla_{L}}(n)
$$

where $a(n)$ is the adaptation step size given by (23), with $a, A$ and $\alpha$ being arbitrary nonnegative parameters:

$$
a(n)=\frac{a}{(A+n+1)^{\alpha}}
$$

A second update is then applied to vector $\underline{X}$ based on the gradient of the DD cost function $J$. Function $J$ measures the mean squared error (MSE) with respect to the reference constellation over the last $N b \leq N_{L}$ samples stored in buffer $\underline{r}(n)=\left[\begin{array}{llll}r\left(n-N_{L}+1\right) & \cdots & r(n-1) & r(n)\end{array}\right]^{T}$ and is given by

$$
J(n)=\frac{1}{N b} \sum_{j=0}^{N b-1}(|Q\{r(n-j)\}-r(n-j)|)^{2}
$$

where $Q\{\cdot\}$ is the operator which returns the reference constellation IQ symbol with the smallest Euclidean distance to the argument.

This second update of vector $\underline{X}$, given by (25), is a conditional update based on the control variable $D$, which depends on the same closeness of the output $r(n)$ and the perturbed output $r^{\mathrm{I}}(n)$ to the same IQ symbol in the reference constellation [3]. Notice that the perturbation here is implicitly obtained from the first update of vector $\underline{X}$.

$$
\underline{X}(n+1)=\underline{X^{\mathrm{II}}}(n)=\underline{X^{\mathrm{I}}}(n)-(1-D) b(n) \underline{\nabla_{J}}(n)
$$

where $D=0$ if $Q\{r(n)\}=Q\left\{r^{\mathrm{I}}(n)\right\}$ and $D=1$ if 
$Q\{r(n)\} \neq Q\left\{r^{\mathrm{I}}(n)\right\}, \quad$ with $r(n)$ being the array output signal for reactance vector $\underline{X}(n)$ and $r^{\mathrm{I}}(n)$ being the array output signal for reactance vector $\underline{X^{\mathrm{I}}}(n)$. Adaptation step size $b(n)$ is given by (26), with $b, B$ and $\beta$ being arbitrary nonnegative parameters:

$$
b(n)=\frac{b}{(B+n+1)^{\beta}}
$$

and the gradient $\underline{\nabla_{J}}$ of the cost function $J$ is given by

$$
\underline{\nabla_{J}}=\frac{\Delta J}{\Delta \underline{X}}=\frac{J(\underline{X})-J\left(\underline{X^{\mathrm{I}}}\right)}{\underline{X}-\underline{X^{\mathrm{I}}}}
$$

Table 1 shows the step-by-step procedure for the concurrent MMC-DD beamformer.

TABLE I

\begin{tabular}{|c|c|}
\hline Step & Procedure \\
\hline 1 & $\begin{array}{l}\text { Initialize reactance vector: } \\
\underline{X}=\left[\begin{array}{lllllll}50 & 0 & 0 & 0 & 0 & 0 & 0\end{array}\right]^{T} \\
\begin{array}{l}\text { Initialize received IQ symbol (iteration) } \\
n=0\end{array}\end{array}$ \\
\hline 2 & Determine $\underline{w}=(\mathbf{Z}+\underline{X})^{-1} \underline{U}$ from (8). \\
\hline 3 & $\begin{array}{l}\text { Initialize } \\
\underline{r}(n)=\left[\begin{array}{llll}r\left(n-N_{L}+1\right) & \cdots & r(n-1) & r(n)\end{array}\right]^{T} \\
\text { with } N_{L} \text { samples of the output signal } r(n) \text { obtained from (1)-(4), } \\
\text { with } n=0,1 \cdots, N_{L}-1 \text {, being } N_{L} \geq N a \text { and } N_{L} \geq N b .\end{array}$ \\
\hline 4 & \begin{tabular}{lllllll} 
Generate & \multicolumn{1}{c}{ random } & sequence \\
$\Delta=$ & $=\left[\begin{array}{lllllll}0 & \delta_{1} & \delta_{2} & \delta_{3} & \delta_{4} & \delta_{5} & \delta_{6}\end{array}\right]^{T}$
\end{tabular} \\
\hline 5 & $\begin{array}{l}\text { Determine } a(n), b(n) \text { e } c(n) \text { according to (23), (26) and } \\
\text { (15). }\end{array}$ \\
\hline 6 & Determine $\underline{X}_{\text {up }}$ and $\underline{X}_{\text {down }}$ according to (13) and (14). \\
\hline 7 & Obtain $\underline{w}_{\text {up }}$ and $\underline{w}_{\text {down }}$ from (8) by using $\underline{X}_{\text {up }}$ and $\underline{X}_{\text {down }}$. \\
\hline 8 & $\begin{array}{l}\text { Obtain } r_{\text {up }}(n) \text { and } r_{\text {down }}(n) \text { from (1)-(4) by using } \underline{w}_{\text {up }} \text { and } \\
\underline{w}_{\text {down }} \text {. }\end{array}$ \\
\hline 9 & $\begin{array}{l}\text { Store } r_{\text {up }}(n) \text { and } r_{\text {down }}(n) \text { respectively in vectors } \\
\underline{r}_{\text {up }}(n)=\left[\begin{array}{lllll}r\left(n-N_{L}+1\right) & \cdots & r(n-1) & r_{\text {up }}(n)\end{array}\right]^{T} \\
\text { and } \\
\underline{r}_{\text {down }}(n)=\left[\begin{array}{lllll}r\left(n-N_{L}+1\right) & \cdots & r(n-1) & r_{\text {down }}(n)\end{array}\right]^{T} .\end{array}$ \\
\hline 10 & $\begin{array}{l}\text { Obtain } L_{\text {up }} \text { and } L_{\text {down }} \text { from (16) by using } \underline{r}_{\text {up }}(n) \text { and } \\
\underline{r}_{\text {down }}(n) \text {. }\end{array}$ \\
\hline 11 & Determine gradient $\underline{\nabla_{L}}$ from (20) and (21). \\
\hline 12 & Determine $\underline{X^{\mathrm{I}}}(n)$ from (22). \\
\hline 13 & ine $w^{I}=$ \\
\hline
\end{tabular}

STEP-BY-STEP PROCEDURE FOR THE CONCURRENT MMC-DD BEAMFORMER

\begin{tabular}{|c|l|}
\hline 14 & Obtain $r^{\mathrm{I}}(n)$ from (1)-(4) by using $\underline{w^{\mathrm{I}}}$. \\
\hline 15 & $\begin{array}{l}\text { Determine conditional update control variable: } D=0 \quad \text { if } \\
Q\{r(n)\}=Q\left\{r^{\mathrm{I}}(n)\right\} \text { and } D=1 \text { if } Q\{r(n)\} \neq Q\left\{r^{\mathrm{I}}(n)\right\} .\end{array}$ \\
\hline 16 & Determine $\underline{\nabla_{J}}$ from (27). \\
\hline 17 & Determine $\underline{X}(n+1)=\underline{X^{\mathrm{II}}}(n)$ from (25). \\
\hline 18 & Move one position left all samples stored in buffer $\underline{r}(n)$. \\
\hline 19 & Determine $\underline{w^{\mathrm{II}}=\left(Z+\underline{X^{\mathrm{II}}}\right)^{-1} \underline{U} \text { from }(8) .}$ \\
\hline 20 & Obtain $r^{\mathrm{II}}(n)$ from (1)-(4) by using $\underline{w^{\mathrm{II}}}$. \\
\hline 21 & $\begin{array}{l}\text { Store } r^{\mathrm{II}}(n) \text { in vector } \\
\underline{r}(n)=\left[r\left(n-N_{L}+1\right) \quad \cdots \quad r(n-1) \quad r^{\mathrm{II}}(n)\right]^{r} .\end{array}$ \\
\hline 22 & $\begin{array}{l}n=n+1 \\
\text { Go to step } 4 .\end{array}$ \\
\hline
\end{tabular}

\section{SIMULATION RESULTS}

In order to assess the performance of the MMC-DD concurrent beamformer we present simulation results for the case of one interfering path and for the case of two interfering paths. The signals received from these interfering paths are delayed versions of the main signal.

The adopted beamformer parameters are: $a=4000000$, $b=4000000, \alpha=0.6, \beta=0.6, \gamma=0.15, A=200$, $B=250, \quad C=0.05, \quad N a=50$ e $N b=30$. These parameters were experimentally obtained as a compromise between convergence rate and steady state MSE. System modulation is 16-QAM with symbol rate $10.2 \mathrm{MHz}$. The beamformer is considered to converge at the $n^{\text {th }}$ iteration if the MSE with respect to the reference constellation attains a value lower than 0.001 .

Referring to Figures 2 and 3, the main and interfering paths impinge the array at $\theta=90^{\circ}$ and $\phi$ as specified below:

Case I: Main path impinges the array at $\phi=0^{\circ}$ and one interfering path impinges the array at $\phi=90^{\circ}$. Interfering path signal is the main path signal attenuated by $13.8 \mathrm{~dB}$ and delayed by $0.15 \mu \mathrm{s}$.

Case II: Main path impinges the array at $\phi=0^{\circ}$ and two interfering paths impinge the array at $\phi=90^{\circ}$ and $\phi=180^{\circ}$. Interfering path 1 signal is the main path signal attenuated by $13.8 \mathrm{~dB}$ and delayed by $0.15 \mu \mathrm{s}$. Interfering path 2 signal is the main path signal attenuated by $16.2 \mathrm{~dB}$ and delayed by 2.22 $\mu \mathrm{s}$.

Figure 4 compares the output signal constellation after convergence for the MMC beamformer and for the MMC-DD concurrent beamformer. Notice that the MMC-DD beamformer is able to correct the constellation phase offset of the output signal $r$. 


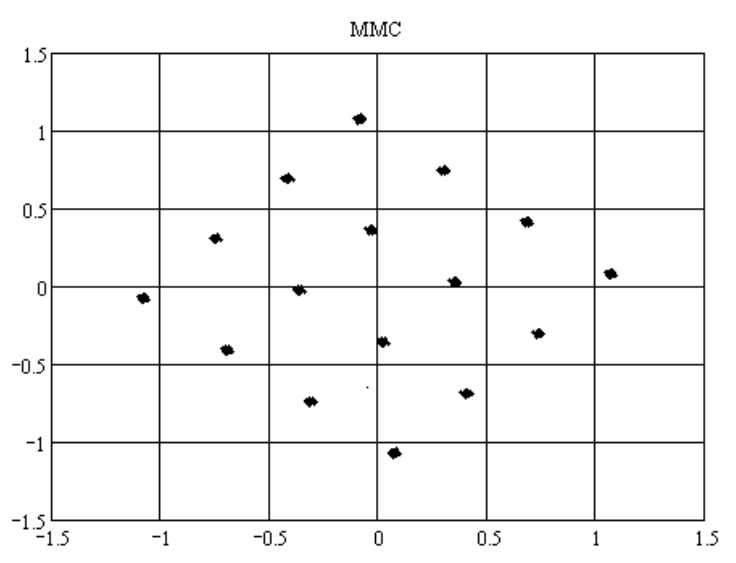

Fig. 4(a) Output signal constellation after MMC convergence for Case I.

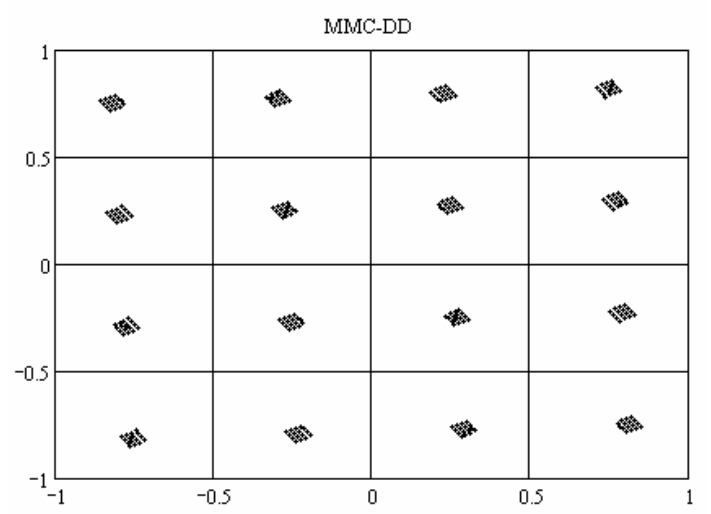

Fig. 4(b) Output signal constellation after MMC-DD convergence for Case I.

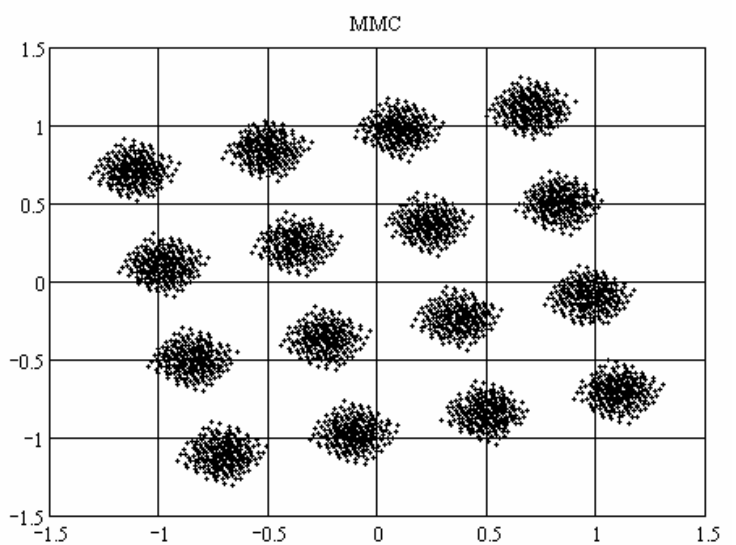

Fig. 4(c) Output signal constellation after MMC convergence for Case II.

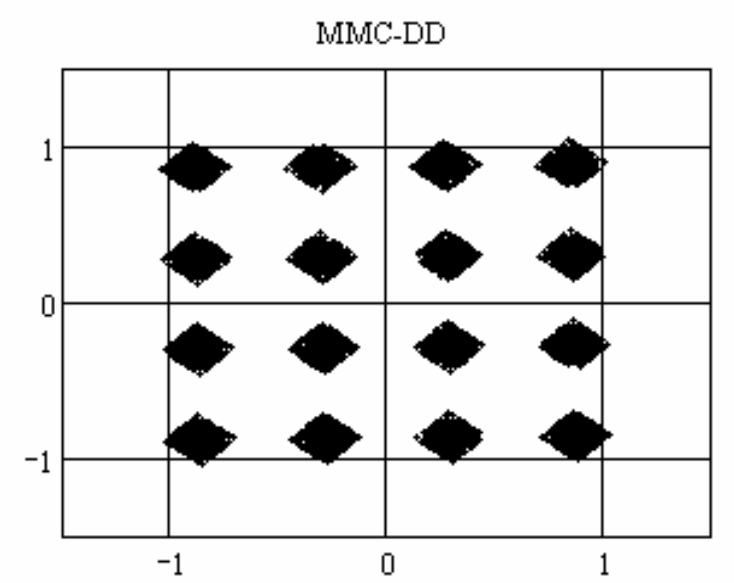

Fig. 4(d) Output signal constellation after MMC-DD convergence for Case II.

Figure 5 shows the symbol error rate (SER) at the demodulator output for the MMC and MMC-DD beamformers operating under Cases I and II conditions. These SER values are obtained with no channel compensation except that performed by the beamformer (i.e., time equalizer is off), which means the SER would be even lower if the time equalizer were on. Notice the lower SER of the MMC-DD beamformer as the SNR increases.

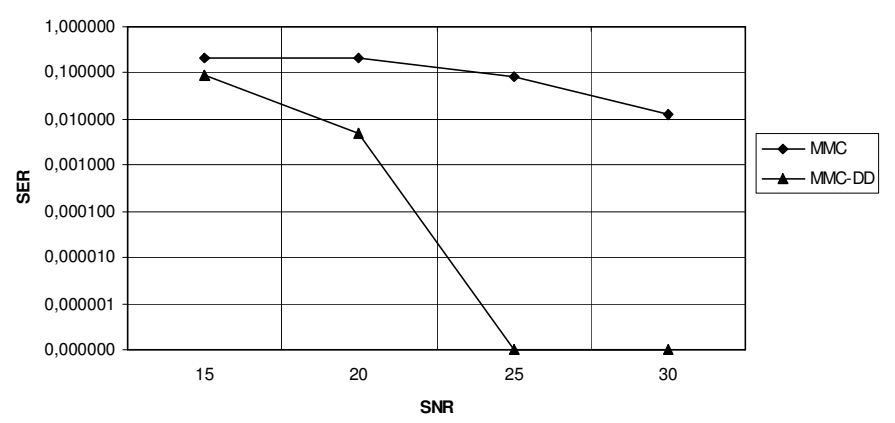

Fig. 5(a) Demodulator SER for Case I.

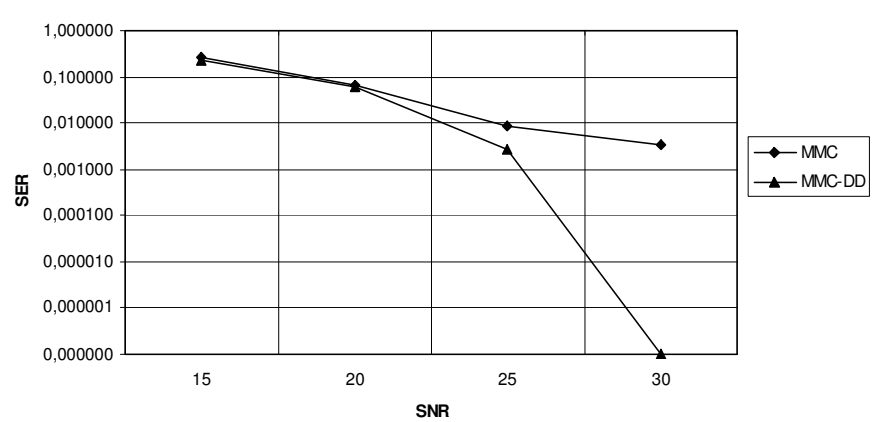

Figure 5(b) Demodulator SER for Case II.

Figure 6 shows the MSE curves of output signal $r$ with respect to the reference constellation versus received IQ symbol index $n$. Notice the faster convergence and the lower final MSE for the MMC-DD beamformer in comparison with the MMC beamformer. 


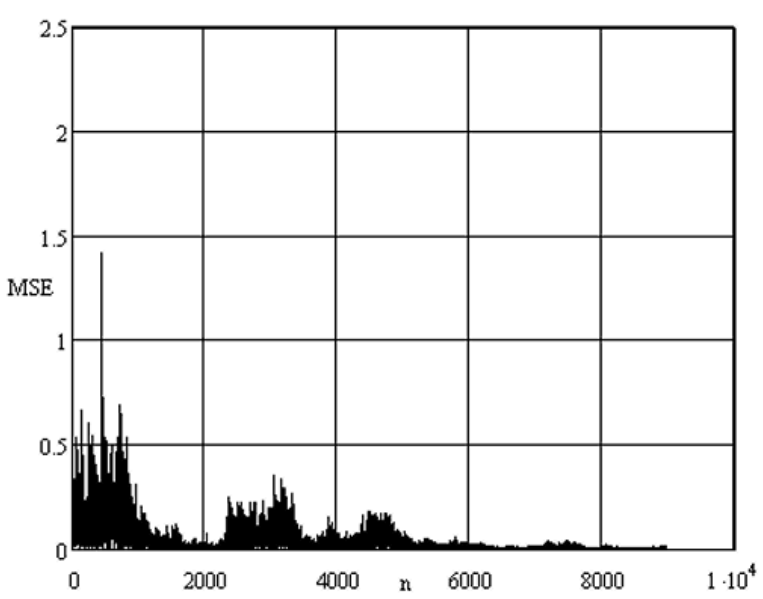

Fig. 6(a) MSE for the MMC beamformer - Case I.

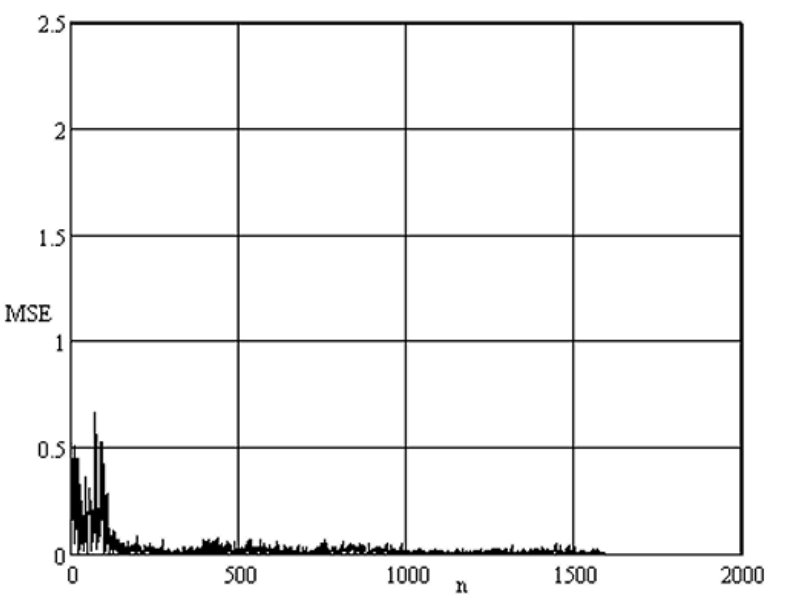

Fig. 6(b) MSE for the MMC-DD beamformer - Case I.

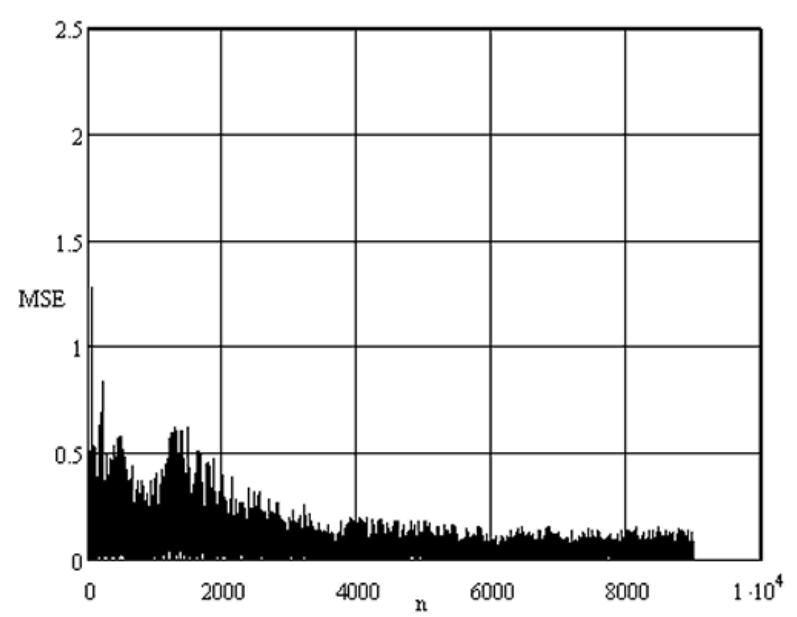

Fig. 6(c) MSE for the MMC beamformer - Case II.

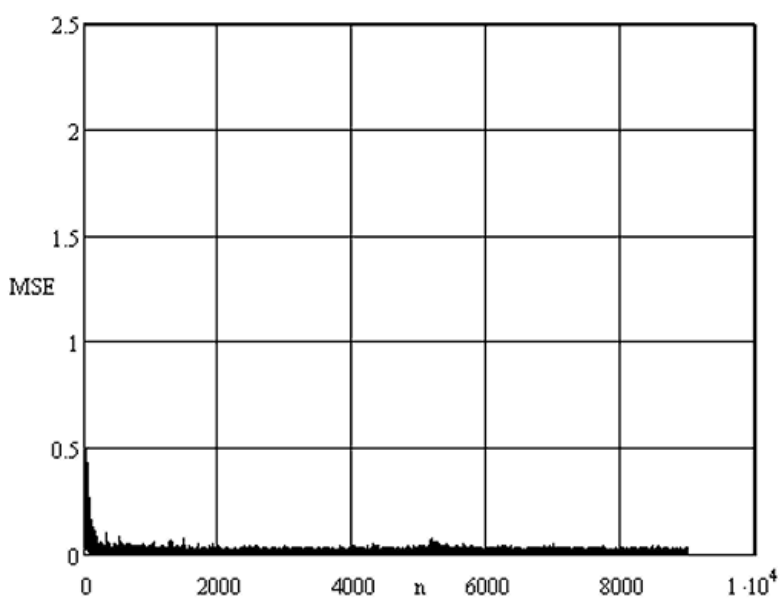

Fig. 6(d) MSE for the MMC-DD beamformer - Case II.

Figure 7 shows the radiation pattern after the algorithm convergence. Notice that the concurrent MMC-DD beamformer presents a better signal to interference ratio than the MMC approach.

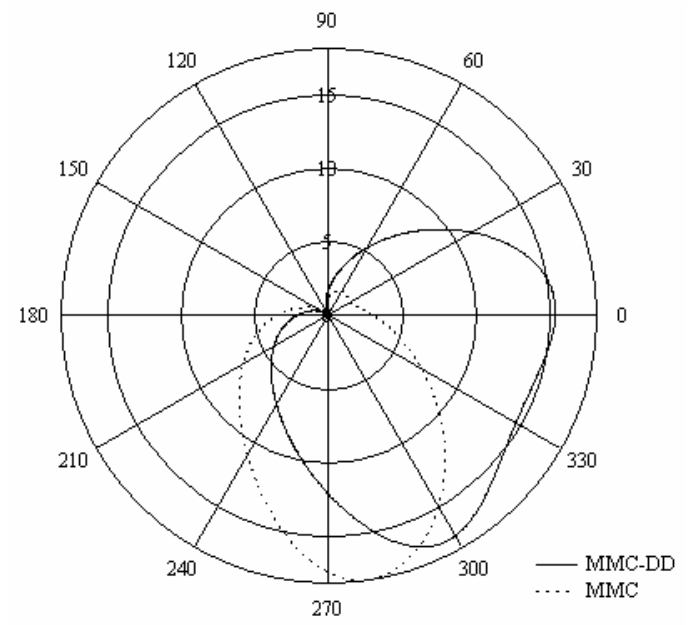

Fig. 7(a) Array radiation pattern for the MMC and MMC-DD beamformers for Case I.

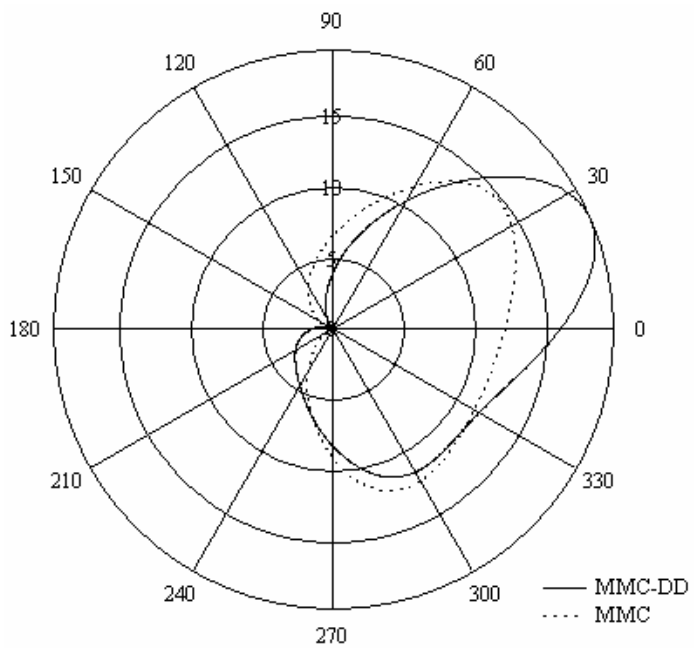

Fig. 7(b) Array radiation pattern for the MMC and MMC-DD beamformers for Case II. 


\section{CONCLUSION}

The concurrent minimization of two cost functions - one that measures energy dispersion and other that measures closeness to the reference constellation - leads to a superior performance regarding to the demodulation symbol error rate. In fact, the symbol error rate for the concurrent MMC-DD beamformer is at least one magnitude order lower than the MMC for signal to noise ratios above $25 \mathrm{~dB}$.

A conditional update based on the certainty of the beamformer output controls the simultaneous concurrent minimization of the two cost functions. Certainty is measured as the same closeness of the non-perturbed and perturbed beamformer outputs to the same IQ symbol in the reference constellation. Results have shown that this new technique is able to attain a significant lower mean squared error with respect to the system reference constellation with a much faster convergence rate.

The new beamformer presented in this article is suitable to be applied to most wireless digital systems including emerging technologies, such as IEEE 802.16e, among others.

\section{REFERENCES}

[1] K. Yang and T. Ohira , "Realization of Space-Time Adaptive Filtering by Employing Electronically Steerable Passive Array Radiator Antennas", IEEE Transactions on Antennas and Propagation, Vol. 51, No. 7, July 2003.

[2] C. Sun, A. Hirata, T. Ohira and N. C. Karmakar, "Fast Beamforming of Electronically Steerable Parasitic Array Radiator Antennas: Theory and Experiment", IEEE Transactions on Antennas and Propagation, Vol. 52, No. 7, July 2004.

[3] Fernando C.C. De Castro, Maria C. F. De Castro and Dalton S. Arantes "Concurrent Blind Deconvolution for Channel Equalization", IEEE International Conference on Communications ICC2001, pp. 366-371, Helsinki, Finland, June 2001.

[4] J.C. Liberti and T.S. Rappaport, Smart Antennas for Wireless Communications, Prentice Hall PTR, 1999.

[5] K.R. Dandekar, H. Ling, and G. Xu, "Experimental Study of Mutual Coupling Compensation in Smart Antenna Applications", IEEE Transactions on Wireless Communications, Vol. 1, No. 3, July 2002.

[6] R.C. Hansen, Moment Methods in Antennas and Scattering, Artech House, 1990.

[7] J. Jin, The Finite Element Method in Electromagnetics, $2^{\text {nd }}$, John Wiley \& Sons, 2002.

[8] A.F. Peterson, S.L. Ray and R. Mittra, Computational Methods for Electromagnetics, IEEE Press, 1998.

[9] C.A. Balanis, Antenna Theory - Analysis and Design, $2^{\text {nd }}$, John Wiley \& Sons, 1997.

[10] W.L. Stutzman and G.A. Thiele, Antenna Theory and Design, $2^{\text {nd }}$, John Wiley \& Sons, 1998.

[11] T. Ohira, "Blind Adaptive Beamforming Electronically-Steerable Parasitic Array Radiator Antenna Based on Maximum Moment Criterion", Antennas and Propagation Society International Symposium, 2002 - IEEE. Vol. 2, 16-21 June 2002.

[12] S. Chen and E.S. Chang, "Fractionally spaced blind equalization with low-complexity concurrent constant modulus algorithm and soft decision-directed scheme", International Journal of Adaptive Control and Signal Processing 19(6) pp. 471-484, 2005.

[13] R.F. Harrington, Field Computation by Moment Methods, IEEE Press, 1993.

[14] J. Moore and R. Pizer, Moment Methods in Electromagnetics, John Wiley \& Sons, 1984.

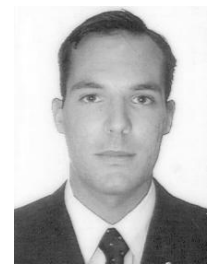

Leonardo Ortolan was born in Guapore, Brazil, on May 15, 1978. He received the B.E. degree from the Department of Electrical Engineering, Pontifical Catholic University of Rio Grande do Sul, Brazil, in 2003, and the M.S. Degree from the Post Graduation in Electrical Engineering Program, Pontifical Catholic University of Rio Grande do Sul, Brazil, in 2007.

Since 2004 he has been engaged in research on Wireless Systems, specially Digital Television at Wireless Technologies Research Center, Pontifical Catholic University of Rio Grande do Sul, Brazil.

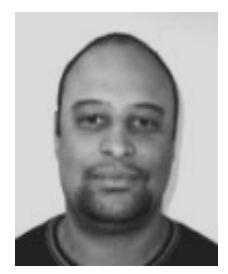

Thiago Luís Silva Santos was born in Porto Alegre, Brazil, on March 25, 1978. He received the B.E. degree from the Department of Electrical Engineering, Pontifical Catholic University of Rio Grande do Sul, Brazil, in 2005.

Since 2005 he has been engaged in research on Wireless Systems, specially Digital Television, at Wireless Technologies Research Center, Pontifical Catholic University of Rio Grande do Sul, Brazil.

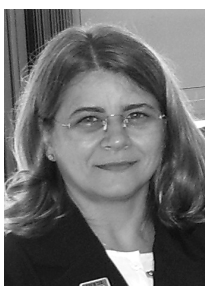

Maria Cristina Felippetto DeCastro was born in Porto Alegre, Brazil, on July 14, 1958. She received the B.E. degree from the Department of Electrical Engineering, Pontifical Catholic University of Rio Grande do Sul, Brazil, in 1983, the M.S. Degree from the Post Graduation in Electrical Engineering Program, Pontifical Catholic University of Rio Grande do Sul, Brazil, in 1996 and the Doctor Degree from the Electrical Engineering and Computation Faculty, Campinas State University, Brazil, in 2001.

Since 1997 she has been engaged in research on Wireless Systems, specially Digital Television, until 2001 at Campinas State University, and after that at Wireless Technologies Research Center, Pontifical Catholic University of Rio Grande do Sul, Brazil. In 2007 she was nominated Director of Engineering Faculty, Pontifical Catholic University of Rio Grande do Sul.

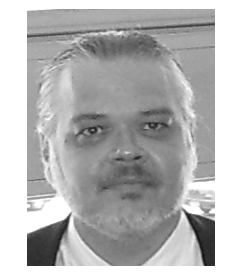

Fernando César Comparsi DeCastro was born in Porto Alegre, Brazil, on November 12, 1959. He received the B.E. degree from the Department of Electrical Engineering, Pontifical Catholic University of Rio Grande do Sul, Brazil, in 1983, the M.S. Degree from the Post Graduation in Electrical Engineering Program, Pontifical Catholic University of Rio Grande do Sul, Brazil, in 1995 and the Doctor Degree from the Electrical Engineering and Computation Faculty, Campinas State University, Brazil, in 2001.

Since 1997 he has been engaged in research on Wireless Systems, specially Digital Television, until 2001 at Campinas State University, and after that at Wireless Technologies Research Center, Pontifical Catholic University of Rio Grande do Sul, Brazil. 